Pensamiento Crítico N. ${ }^{\circ} 14$, pp. 9-25

\title{
Relaciones Económicas China-Japón: Los dos gigantes de Asia cada vez más integrados
}

\author{
Carlos Aquino Rodríguez
}

\section{RESUMEN}

China y Japón, ahora la segunda y tercera economías más grandes del mundo, tienen cada vez una mayor relación comercial producto de que ambos se necesitan. Japón requiere la mano de obra barata china y su inmenso mercado interno, y también ahora sus turistas, y China necesita el capital, la tecnología y el know how empresarial japonés. Japón aprendió muchas cosas de China, aunque a través de la historia las relaciones entre ambos atravesaron periodos complicados. La necesidad mutua hará que estos dos colosos se integren económicamente aún más.

Palabras clave: Relaciones China-Japón, economía china, economía japonesa

\section{SUMMARY}

China and Japan are the second and third largest economies in the world now and continue to increase their economic relationship as they need each other. Japan needs China cheap labor force and its big internal market, while China needs Japan technology, capital and management know how. Japan through history learned many things from China even if during a period both countries encountered difficult times. As they need each other Japan and China will continue to get closer.

Keywords: China-Japan relationship, china economy, japan economy 


\section{Carlos Aquino Rodríguez}

El 31 de julio del 2010 se anunció que China ya superó a Japón y se convirtió en la segunda economía más grande del mundo ${ }^{1}$. La economía china creció $11.1 \%$ en el primer semestre del 2010, mientras que la economía japonesa lucha por salir de la recesión de los últimos dos años. En todo caso, como las economías más grandes en el Asia Oriental, Japón y China incrementan más sus relaciones de todo tipo, especialmente las económicas. Y de todas maneras a fines del año 2010 se confirmó que Japón, que era la segunda economía más grande del mundo, ya no lo es, y China, que era la tercera, se ha convertido en la segunda más grande.

La economía japonesa, que estuvo en recesión el 2008 y el 2009, parece recuperarse lentamente gracias, en gran parte, a las exportaciones que hace a China, que crece a tasas de casi 10\% anual (desde 1979, año en que empezó sus reformas económicas que lo abrieron al mundo, China ha crecido a un promedio anual de casi $10 \%$, por 30 años consecutivos). En contraste, Japón ha tenido en las dos últimas décadas un periodo de bajo crecimiento, lo que los japoneses llaman décadas perdidas. En los años 1990s su crecimiento fue en promedio $1.4 \%$ al año, y la década pasada fue de $0.7 \%$ anual, terminando esta en recesión, con un $-1.2 \%$ el 2008 y -5.2\% el 2009. Ver Cuadro 2.

En el presente artículo se analiza las relaciones China-Japón en sus diferentes aspectos. En la Introducción se da una revisión general al comportamiento de sus economías en los últimos años; segundo, se ve el aspecto del Comercio e Inversión; tercero, el movimiento de personas; cuarto, la cooperación económica; quinta, la relación política entre ambos, y por último se dan las conclusiones.

\section{INTRODUCCIÓN}

Como se ve en el Cuadro 1, en los últimos años la economía china ha crecido en forma acelerada, mientras que la de Japón no. La economía china creció hasta convertirse en la tercera más grande en el mundo. El 2009 el tamaño de su PBI fue de 4.9 billones de dólares, detrás de Japón que fue de 5.1 billones de dólares. Ese año, el PBI de EE.UU. fue de 14.4 billones de dólares, la mayor en el mundo. Pero lo más espectacular de China es el crecimiento de sus exportaciones, el motor principal del desarrollo de su economía, que aumento por ejemplo del 2001 al 2008 desde 266 mil millones de

1 "China overtakes Japan as N.o 2 economy: FX chief”, http://www.chinadaily.com.cn/china/2010-07/31/ content_11076255.htm 


\section{Relaciones Económicas China-Japón: Los dos gigantes de Asia cada vez más integrados}

dólares hasta 1.42 billones de dólares, 5.36 veces más en ese lapso, para constituirse el 2008 en el exportador más grande del mundo (El 2009, por la recesión en la economía mundial, las exportaciones de ambos países decrecieron). El año 2004 había pasado a Japón en el monto de exportaciones.

El otro aspecto impresionante de la economía china es el aumento en sus Reservas Internacionales Netas (RIN), que pasó de 212 mil millones de dólares en el 2001 a 2.422 billones de dólares en el 2009. En este aspecto, el año 2006 sobrepaso a Japón para convertirse en el país con la mayor cantidad de RIN en el mundo.

La población china es diez veces más grande que la de Japón, y su territorio, de 9.6 millones de kilómetros cuadrados, es 25 veces más grande que el japonés, de solo 378 mil kilómetros cuadrados. La población china continúa creciendo, aunque a un tasa menor a $1 \%$ al año, por la política de un niño por familia, implantado desde las reformas económicas que abrieron a China al mundo hace treinta años, mientras que la población japonesa prácticamente no crece, desde hace cuatro años ${ }^{2}$. En el 2009 Japón tuvo un PBI per cápita de 40220 dólares, más de once veces mayor que el de China, que fue de 3619 dólares.

Si bien es cierto que el Fondo Monetario Internacional proyectó que la economía japonesa crecería $2.4 \%$ en el año 2010 y $1.8 \%$ en el 2011, mientras que China crecería $10.5 \%$ y $9.6 \%$ en esos años (ver Cuadro $2^{3}$ ), algunos creen que hay la posibilidad de que Japón pueda tener en la presente década otros diez años más de bajo crecimiento económico. Uno de los factores es que Japón sufre un problema de deflación, donde los precios caen, y este fenómeno ha acompañado a este país durante varios periodos de las dos décadas pasadas. Por lo pronto esta tendencia continuará el 2010, y es probable también el 2011. Por ejemplo, en junio del 2010 el índice de precios al consumidor cayó otra vez por 16 meses consecutivos en el país 4 .

2 La población de Japón al 31 de marzo del 2010 alcanzó los 127057 860, menor en 18323 que el año anterior. El 2008 y 2009 subió, pues el número de japoneses que regresaron al país fue mayor al declive natural de la población. Ver "Japan population's falls for 1st time in three years", http://www.breitbart.com/ article.php?id=D9H9U8EG0\&show article $=1$

3 Ver el informe de julio del 2010 sobre la evolución de la economía mundial por el FMI: "World Recovery Continues, But Risks Increase, Say IMF", http://www.imf.org/external/pubs/ft/survey/so/2010/RES070710A.htm

4 "Japan's unemployment rises, output falls", http://au.biz.yahoo.com/100730/33/2ep2n.html\#Scene_1 


\section{Carlos Aquino Rodríguez}

Cuadro 1. Indicadores económicos, China y Japón (en miles de millones de dólares).

\begin{tabular}{|c|c|c|c|c|c|c|c|c|c|}
\hline Años & $\mathbf{2 0 0 1}$ & $\mathbf{2 0 0 2}$ & $\mathbf{2 0 0 3}$ & $\mathbf{2 0 0 4}$ & $\mathbf{2 0 0 5}$ & $\mathbf{2 0 0 6}$ & $\mathbf{2 0 0 7}$ & $\mathbf{2 0 0 8}$ & $\mathbf{2 0 0 9}$ \\
\hline $\begin{array}{c}\text { Exportaciones } \\
\text { China } \\
\text { Japón }\end{array}$ & 266 & 325 & 438 & 593 & 762 & 968 & 1218 & 1428 & 1204 \\
\hline $\begin{array}{c}\text { Importaciones } \\
\text { China } \\
\text { Japón }\end{array}$ & 243 & 295 & 412 & 561 & 660 & 791 & 956 & 1133 & 954 \\
\hline $\begin{array}{c}\text { RIN } \\
\text { China }\end{array}$ & 212 & 286 & 403 & 609 & 818 & 1066 & 1528 & 1946 & 2422 \\
Japón & 395 & 461 & 663 & 824 & 828 & 874 & 948 & 1,003 & 1,024 \\
\hline $\begin{array}{c}\text { Crecimiento } \\
\text { anual PBI (\% } \\
\text { anual) } \\
\begin{array}{c}\text { China } \\
\text { Japón }\end{array}\end{array}$ & 7.5 & 9.1 & 10.0 & 10.11 & 10.40 & 11.61 & 13.01 & 9.05 & 8.7 \\
\hline $\begin{array}{c}\text { Tamaño PBI } \\
\text { China } \\
\text { Japón }\end{array}$ & 0.2 & 0.3 & 1.4 & 2.74 & 1.93 & 2.04 & 2.39 & -0.64 & -5.3 \\
\hline $\begin{array}{c}\text { Población, } \\
\text { millones (mitad } \\
\text { de año) } \\
\text { China } \\
\text { Japón }\end{array}$ & 1276 & 1284 & 1292 & 1299 & 1307 & 1314 & 1321 & 1328 & 1334 \\
\hline
\end{tabular}

Fuente: China Statistical Yearbook, años 2009, 2007, 2003, http://www.stats.gov.cn/tjsj/ndsj/2009/indexeh.htm

* Fuente: CIA: The World Factbook, https:/www.cia.gov/library/publications/the-world-factbook/

Un aspecto que explica este bajo crecimiento económico, y la continua deflación que sufre Japón, es que aquello es el resultado del shock que sufrió este país desde que su moneda empezó a devaluarse en 1985. Japón es un país dependiente de las exportaciones. El valor del yen pasó de 250 yenes por dólar en 1985 a 120 yenes por dólar en 1989 y llegó a tener en abril del 1995 un valor de 80 yenes por dólar. Su economía sufrió un fuerte golpe, cuya secuela aún se siente ${ }^{5}$. Como se ve en el Gráfico 1 , en la segunda mitad de la década pasada su moneda se mantuvo en un promedio de 110 a 120 yenes por dólar; pero desde el 2009 se ha revaluado otra vez y estuvo en el nivel de 85

5 Ver Carlos Aquino: “¿Puede Japón ser otra Grecia? El peso de la deuda pública y los problemas de la economía japonesa". 


\section{Relaciones Económicas China-Japón: Los dos gigantes de Asia cada vez más integrados}

yenes por dólar en la segunda semana de agosto del 2010, con lo que sus exportaciones sufrieron de pérdida de competitividad.

Cuadro 2. Evolución de la economía mundial y proyecciones de crecimiento.

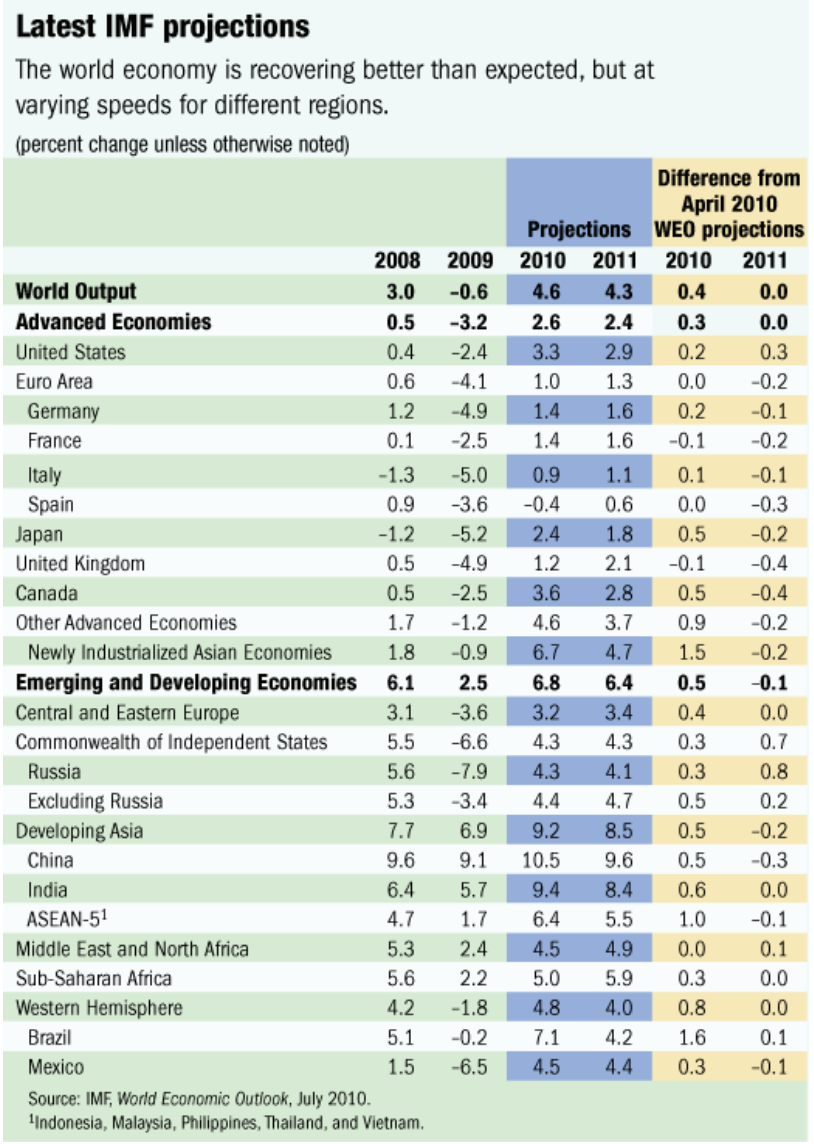

Fuente: http://www.imf.org/external/pubs/ft/survey/so/2010/RES070710A.htm

China, por otro lado, después de devaluar su moneda en 1995 de 5.4 yuanes por dólar a 8.2 yuanes por dólar, lo mantuvo en este valor hasta mediados del 2005, en que permitió que su moneda se revalúe lentamente, hasta alcanzar un valor de 6.8 yuanes 


\section{Carlos Aquino Rodríguez}

Gráfico 1. Tipo de cambio, yenes por dólar.

\section{USD/JPY}

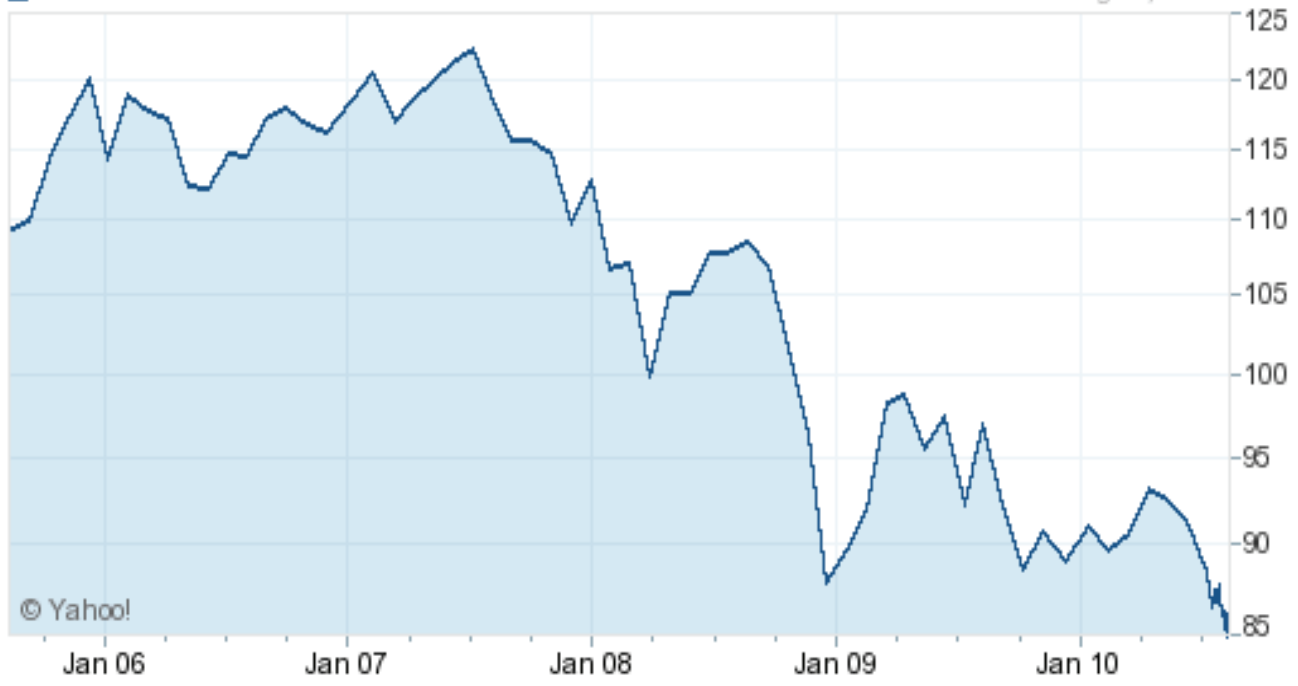

Fuente: http://finance.yahoo.com/q/bc?s=USDJPY $=\mathrm{X} \& \mathrm{t}=5 \mathrm{y} \& \mathrm{l}=\mathrm{on} \& \mathrm{z}=\mathrm{m} \& \mathrm{q}=1 \& \mathrm{c}=$

por dólar a mediados del 2008. Pero desde ese momento lo mantuvo casi fijo. Ante las acusaciones de que China mantenía un valor bajo del yuan para favorecer sus exportaciones, y por los crecientes superávit comerciales que China tenía con sus socios comerciales $^{6}$, el gobierno el 19 de junio del 2010 decidió flexibilizar el valor de su moneda, pero como se ve en el Gráfico 2, su valor casi no ha cambiado desde entonces.

Por último, en el aspecto político, Japón está en un periodo de cambios después de tener por casi 54 años, desde 1955, el dominio absoluto de un partido político, el Partido Democrático Liberal (Jiminto en idioma japonés). En setiembre del 2009 ingresó un nuevo gobierno, del Partido Democrático (Minshuto en idioma japonés) ${ }^{7}$, que salió debilitado en las elecciones de julio del 2010 para renovar la mitad de la Cámara de Senadores. Este partido perdió la mayoría en esta Cámara, y tendrá elecciones en se-

6 Ver Carlos Aquino: "La reevaluación del yuan y sus efectos".

7 Ver Carlos Aquino: "Cambio de Gobierno en Japón y sus efectos en la economía". 


\section{Relaciones Económicas China-Japón: Los dos gigantes de Asia cada vez más integrados}

Gráfico 2. Tipo de cambio, yuanes por dólar.

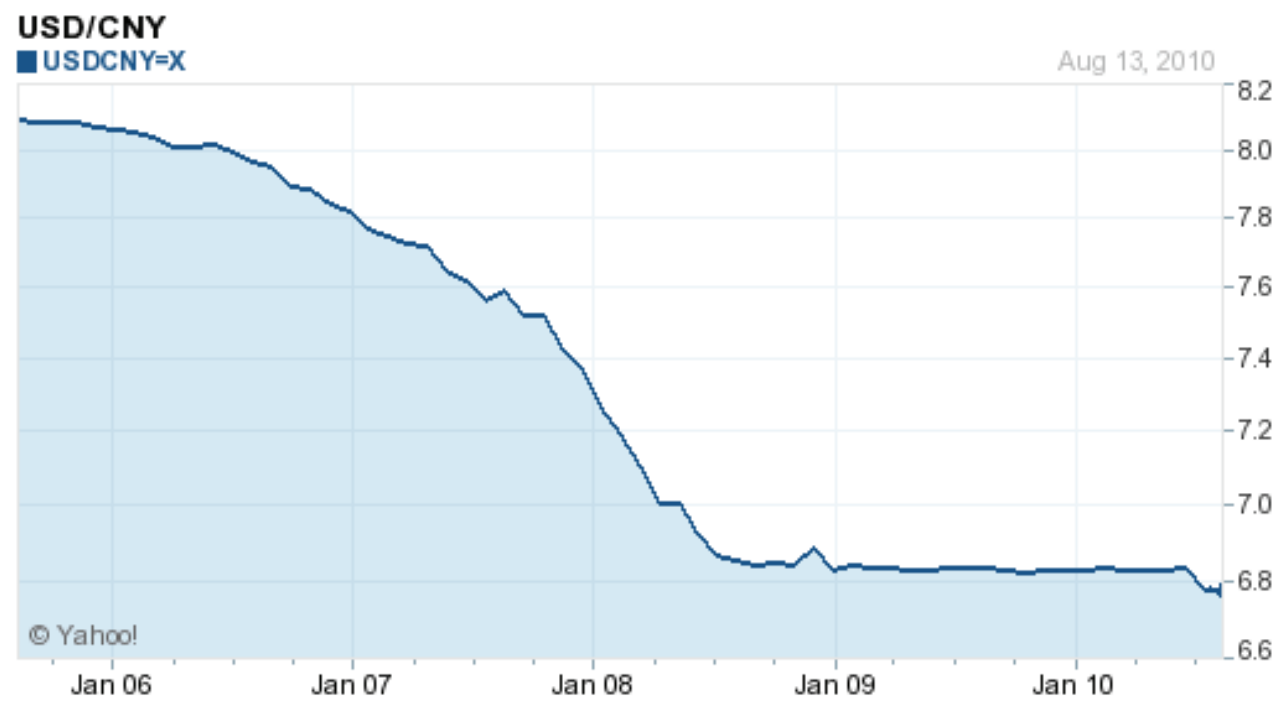

Fuente: $\mathrm{http}: / /$ finance. $y$ ahoo.com/q/bc?s $=\mathrm{USDCNY}=\mathrm{X} \& \mathrm{t}=5 \mathrm{y} \& \mathrm{l}=\mathrm{on} \& \mathrm{z}=\mathrm{m} \& \mathrm{q}=1 \& \mathrm{c}=$

tiembre del 2010 para elegir un nuevo Secretario General (que se convertiría en Primer Ministro pues el partido gobernante tiene aún mayoría en la Cámara de Diputados, y en Japón el Congreso elige al Primer Ministro). Si el presente Primer Ministro es cambiado, sería uno de los tantos que ha tenido Japón en pocos años. Japón ha tenido ya seis primeros ministros en los últimos cuatro años.

Las cosas en Japón se presentan complicadas y un impasse en su clase política ${ }^{8}$ no ayudará a tomar medidas para reactivar su economía, dependiente de las exportaciones, asediada por la deflación y con una enorme deuda pública, la mayor en el mundo como porcentaje del tamaño de su economía (200\% del PBI).

Por otro lado, en China el Partido Comunista tiene un monopolio del poder, lo que le permite tomar las decisiones rápidamente y sin oposición.

8 "A Clouded Outlook" http://www.time.com/time/magazine/article/0,9171,2005689,00.html?xid=rss-topstories 


\section{Carlos Aquino Rodríguez}

\section{Comercio e inversión}

El comercio total entre China y Japón el 2009 fue de 228.85 mil millones de dólares. Las exportaciones chinas a Japón representaron en el 2009 el 10.36\% del total de ese país, menos que el $21 \%$ del total que alcanzó en 2006. Japón fue el tercer socio comercial más importante de China el 2009, pero China fue el socio comercial más importante de Japón ese año (según datos de China) ${ }^{9}$. En todo caso el comercio con China representa ya para Japón una quinta parte del comercio que efectúa con el mundo, y se incrementa conforme pasan los años. En el Cuadro 3 se confirma que China es ya el socio comercial más importante de Japón, sobrepasando a EE.UU.

Cuadro 3. Comercio e Inversión de Japón con China (en miles de millones de dólares)

\begin{tabular}{|c|c|c|c|c|}
\hline Años & $\begin{array}{c}\text { Exportaciones } \\
\text { de Japón a } \\
\text { China (y \% del } \\
\text { total japonés) }\end{array}$ & $\begin{array}{c}\text { Importaciones } \\
\text { de Japón desde } \\
\text { China (y \% del } \\
\text { total japonés) }\end{array}$ & $\begin{array}{c}\text { IED de Japón a } \\
\text { China (flujo, en } \\
\text { base a cifras de } \\
\text { la Balanza de } \\
\text { Pagos) }\end{array}$ & $\begin{array}{c}\text { IED de China a } \\
\text { Japón (flujo, en } \\
\text { base a cifras de la } \\
\text { Balanza de Pagos) }\end{array}$ \\
\hline 2004 & $73.8(13.1)$ & $94.2(20.7)$ & 5.8 & -0.009 \\
\hline 2005 & $80.3(13.4)$ & $109.1(21)$ & 6.5 & 0.011 \\
\hline 2006 & $92.8(14.3)$ & $118.5(20.5)$ & 6.1 & 0.012 \\
\hline 2007 & $109.0(15.3)$ & $127.6(20.55)$ & 6.2 & 0.015 \\
\hline 2008 & $124.0(16.0)$ & $142.3(18.8)$ & 6.4 & 0.037 \\
\hline 2009 & $109.6(18.9)$ & $122.5(22.2)$ & 6.8 & -0.137 \\
\hline $2010 *$ & $56.3(18.9)$ & $57.3(21.4)$ & & \\
\hline
\end{tabular}

* Enero a Mayo. Para el comercio ver Japan's International Trade in Goods (Monthly) y para inversión ver FDI flow (Based on Balance of Payments, net), http://www.jetro.go.jp/en/reports/statistics/

Como se ve en el Cuadro 3, año a año la importancia de China aumenta para Japón. China es utilizada por las compañías japonesas como base para producir para el mundo, y parte de esa producción, en forma creciente, es llevada al mercado japonés. Las tiendas de 100 yenes (de 1.17 dólares al cambio actual) que existen en Japón, que ofrecen una gran variedad de productos a ese precio, muy baratos, donde la inmensa 9 "China-Japan economic ties still tight", http://news.xinhuanet.com/english2010/video/2010-06/01/ c_13326234.htm 


\section{Relaciones Económicas China-Japón: Los dos gigantes de Asia cada vez más integrados}

mayoría de estos vienen de China, es una muestra de la presencia mayor de productos de ese país en Japón. Compañías japonesas como Daiso -que tiene unas 2570 de esas tiendas, el $60 \%$ del total de las tiendas de 100 yenes en Japón- ofrece más de 90 000 productos manufacturados casi todos estos en China ${ }^{10}$.

Por el lado de las inversiones, de acuerdo a estadísticas de Japón, el 2009 este país invirtió 74.6 mil millones de dólares en el exterior en la forma de Inversión Extranjera Directa (IED) y ese año recibió 11.8 mil millones de dólares en IED $^{11}$. La inversión japonesa en China es importante pero es menor que la que hace en EE.UU. (donde invirtió 10.6 mil millones de dólares el 2009, comparado con los 6.8 mil millones que invirtió en China), sin embargo, la inversión de China en Japón es aún insignificante.

Gráfico 3. Número de empresas japonesas adquiridas por empresas de China (y en valor, medido en barras, en miles de millones de yenes).

\section{Dragon rampant}

Number of Japanese firms acquired by Chinese ones

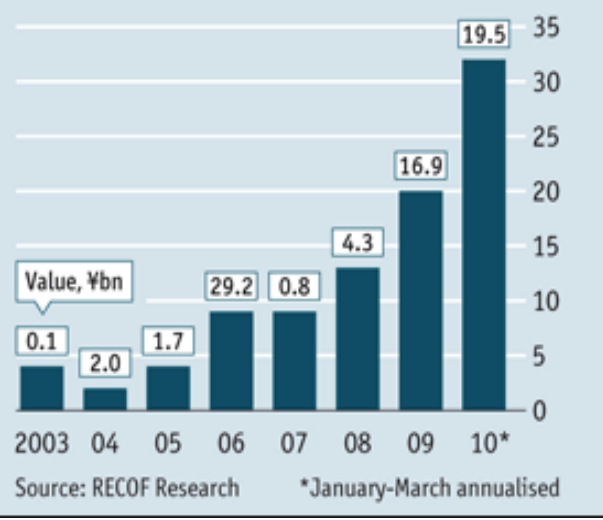

Fuente: http://www.economist.com/node/16010281
Según fuentes de China, a fines del abril del 2010 había más de 43000 compañías con inversión japonesa en ese país, con un monto total invertido de 71000 millones de dólares. Pero China en los últimos años está aumentando su inversión en Japón, y se estima que las empresas chinas han firmado contratos en Japón por más de 14300 millones de dólares (estos datos son de fuente de China) ${ }^{12}$. Compañías de ese país están comprando firmas japonesas, como cuando Laox, una tienda de electrodomésticos japonesa fue comprada por Suning, la compañía de electrodomésticos más grande de China ${ }^{13}$.

Otro renglón donde China está invirtiendo en Japón es en la compra de bonos del gobierno nipón. Solo en los

10 http://www.daiso-sangyo.co.jp/english/products/

11 FDI flow (Based on Balance of Payments, net), http://www.jetro.go.jp/en/reports/statistics/

12 "China, Japan to further promote investment", http://news.xinhuanet.com/english2010/video/2010-06/09/c_13341222.htm

13 "Scaring the salarymen: Chinese firms by Japanese ones", http://www.economist.com/node/16010281 


\section{Carlos Aquino Rodríguez}

primeros meses del año 2010 China compró 1.7 billones de yenes de deuda japonesa (unos 19.9 mil millones de dólares), que es mayor que el monto récord de 255.7 mil millones de yenes que compró en todo el $2005^{14}$. Como se sabe, China está invirtiendo parte de su enorme reserva internacional de moneda extranjera, de casi 2.5 billones de dólares, en activos extranjeros. Ya tiene 867.7 mil millones dólares en deuda del tesoro de EE.UU. (monto algo menor al que tenía a fines del 2009 de 894.8 mil millones de dólares). China está diversificando la tenencia de sus acreencias en dólares, especialmente ahora que el dólar se depreciará frente al yuan en el futuro.

\section{Movimiento de personas}

Por otro lado, Japón necesita los turistas chinos para que dinamicen su economía, ya que al tener una población que decrece necesita revitalizarse con los chinos ricos que viajan por el mundo. Cada vez hay más turistas chinos (los chinos ricos) en Japón,

Gráfico 4. Turistas y trabajadores (practicantes) chinos en Japón (en miles, y en \%).

\section{The Chinese invasion}

Chinese tourists and trainees in Japan

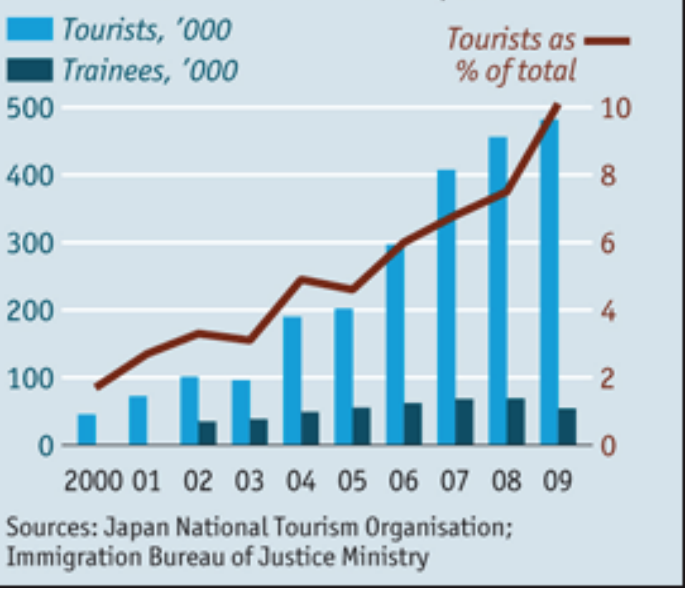

Fuente: http://www.economist.com/node/16542515 que se dice gastan más que cualquier otro turista extranjero en su estadía ahí.

China tiene mucha gente que se ha enriquecido en los últimos años. De acuerdo a la revista Businessweek, ese país, con una población de 1331 millones de habitantes, tenía en el año 2009 unas 670 mil familias que poseen una riqueza de más de un millón de dólares cada una, y que representa el tercer grupo más grande de millonarios del mundo, después de EE.UU. y Japón. Ese número aumentó en un $30.7 \%$ respecto al año anterior, y ellos tienen la mitad

14 "Japan debt is safer than U.S. debt - China economist", http://www.foxbusiness.com/markets/2010/08/11/ japan-debt-safer-debt-china-economist/ 


\section{Relaciones Económicas China-Japón: Los dos gigantes de Asia cada vez más integrados}

de la riqueza del país ${ }^{15}$. Los turistas chinos, que representaban solo el $2 \%$ del total de turistas a Japón el 2000, el año 2009 representaron ya al 10\% (ver Gráfico 4).

Asimismo, en Japón hay una escasez de mano de obra para trabajar en sus fábricas, y desde hace unos años se aceptan trabajadores extranjeros bajo la modalidad de practicantes, y la mayoría de ellos son chinos. Así, como se ve en el Gráfico 4, los chinos, ya sea como turistas ricos o como mano de obra barata, están aumentando en Japón ${ }^{16}$.

Por su parte, los japoneses que viajan a China como turistas han aumentado en los últimos años. En el 2000 viajaron 17.8 millones de japoneses al extranjero, en el 2009 lo hicieron 15.4 millones, pero a China fueron 2.2 millones en el 2000 y 3.3 millones en el 2009. China se está convirtiendo en el destino más popular para los japoneses, y es probable que supere a EE. UU. pronto (En el 2000 fueron 5 millones de japoneses a EE. UU., y en el 2009 fueron 2.9 millones, aunque aparte a Hawai fueron 1.1 millones de japoneses el último año) ${ }^{17}$.

También habría que señalar que hay bastantes estudiantes chinos en Universidades japonesas. Como se ve en el Cuadro 4, casi el $60 \%$ de los estudiantes extranjeros en Japón son de China. Una parte está en becas de su gobierno, otros en becas del gobierno japonés, pero la inmensa mayoría está por sus propios medios. De los 132720 estudiantes extranjeros en Japón al 1 de mayo del 2009, la inmensa mayoría de ellos, 119317 estudiantes, están por sus propios medios ${ }^{18}$.

15 "Countries with the most Millionaires 2010", http://images.businessweek.com/ss/10/06/0615_global_ millionaires/2.htm

16 "Department stores and sweat shops: The Chinese in Japan", http://www.economist.com/ node/16542515

17 "Statistics of Japanese tourists travelling abroad", http://www.tourism.jp/statistics/xls/JTM_outbound20100706.eng.xls

18 "International Students in Japan 2009", http://www.jasso.go.jp/statistics/intl_student/data09_e.html 
Carlos Aquino Rodríguez

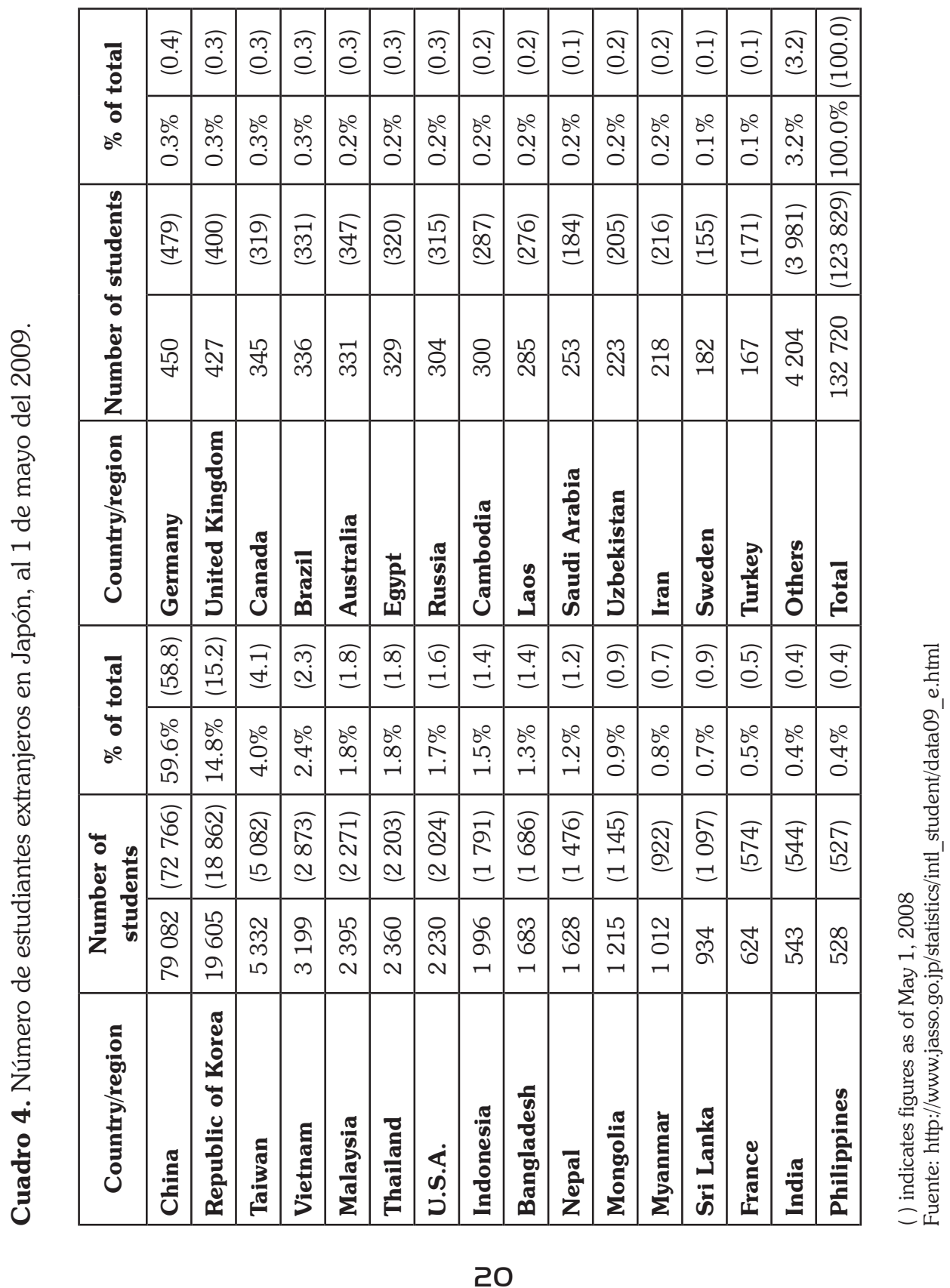




\section{Relaciones Económicas China-Japón: Los dos gigantes de Asia cada vez más integrados}

\section{Cooperación económica}

China es un país en vías de desarrollo que aún recibe ayuda económica, aunque cada vez en menor medida de Japón. Ha habido años en que China fue el principal beneficiario de la Ayuda Oficial para el Desarrollo (AOD) japonesa, y aún se mantiene entre los primeros (ver Cuadro 5), pero el monto que recibe ha disminuido en los últimos años. En el año 2005 fue el segundo que recibió más ayuda del país nipón, con un monto de 1064 millones de dólares, que bajo a 561 millones de dólares el 2006 y 435 millones de dólares el 2007.

El año 2008 China recibió 278.25 millones de dólares en AOD de Japón. Hay que indicar que la AOD incluye préstamos del gobierno (usualmente a tasas de interés bastante menores que de otras fuentes), donaciones (que no hay que devolver), y asistencia técnica. Hasta el 2008 China había recibido de Japón un monto total de desembolso neto de 20826 millones de dólares ${ }^{19}$. De ese monto recibió 14160 millones de dólares

Cuadro 5. Lista de países en los que Japón es el principal donante (en base a desembolso neto, en millones de dólares) (se indica año, monto y porcentaje).

\begin{tabular}{|l|r|r|l|r|r|l|r|r|}
\hline \multicolumn{1}{|c|}{2005} & Amount & Share & \multicolumn{1}{|c|}{2006} & Amount & Share & 2007 & Amount & Share \\
\hline Antigua and Barbuda & 5.42 & 78.3 & Antigua and Barbuda & 1.99 & n.a. & Armenia & 85.23 & 36.9 \\
Indonesia & $1,223.13$ & 54.6 & Viet Nam & 562.73 & 43.1 & Viet Nam & 640.04 & 43.0 \\
Viet Nam & 602.66 & 48.1 & Oman & 1.50 & n.a. & Uzbekistan & 56.32 & 55.0 \\
Uzbekistan & 54.44 & 44.8 & Gambia & 10.99 & 43.8 & Cambodia & 113.56 & 27.2 \\
Oman & 3.72 & n.a. & Cambodia & 106.25 & 30.6 & Kiribati & 13.05 & 58.3 \\
Kazakhstan & 66.17 & 44.8 & Kiribati & 9.88 & 50.5 & Sri Lanka & 44.16 & 14.8 \\
Gambia & 4.38 & 29.0 & Saudi Arabia & 4.61 & 41.3 & Swaziland & 7.26 & 59.9 \\
Cambodia & 100.62 & 29.0 & Samoa & 16.81 & 43.9 & Seychelles & 0.76 & 53.9 \\
Kribati & 11.69 & 54.8 & Sri Lanka & 202.63 & 41.8 & Tanzania & 721.66 & 39.4 \\
Democratic Republic of the Congo & 376.26 & 36.3 & Swaziland & 11.62 & 94.6 & China & 435.66 & 32.7 \\
Samoa & 12.52 & 41.8 & Saint Vincent & 1.38 & 61.1 & Paraguay & 28.90 & 35.0 \\
Sri Lanka & 312.91 & 36.5 & Saint Christopher and Nevis & 4.27 & n.a. & Philippines & 222.16 & 40.6 \\
Swaziland & 25.91 & n.a. & Saint Lucia & 1.95 & 80.2 & Bhutan & 18.07 & 41.7 \\
Saint Vincent & 3.20 & 55.7 & China & 561.08 & 48.1 & Malaysia & 222.97 & n.a. \\
China & $1,064.27$ & 62.9 & Tuvalu & 8.28 & 65.2 & Myanmar & 30.52 & 23.6 \\
Trinidad and Tobago & 1.97 & 32.5 & Trinidad and Tobago & 1.33 & 33.0 & Mongolia & 51.55 & 36.7 \\
Tonga & 11.24 & 45.4 & Paraguay & 25.92 & 41.7 & Laos & 81.46 & 36.7
\end{tabular}

Fuente: Ministry of Foreign Affairs of Japan: "Japan's ODA White Paper 2009", Capítulo 2, Cuadro III-16 (lista parcial), http://www.mofa.go.jp/policy/oda/white/2009/pdfs/part3-2.pdf

19 Ver el documento en idioma japonés "Seifu kaihatsu enjo (ODA) Kokubetsu deta bukku 2009", http:// www.mofa.go.jp/mofaj/gaiko/oda/shiryo/kuni/09 databook/pdfs/01-04.pdf 


\section{Carlos Aquino Rodríguez}

en préstamos, 1163 millones en donaciones, y 5503 millones de dólares en asistencia técnica.

Tradicionalmente, la cooperación japonesa ha estado enfocada en construcción de la infraestructura del país, pero las prioridades cambian con el tiempo, y en los últimos años se ha puesto énfasis en temas como la reducción de la contaminación ambiental, que no solo afecta a China, sino cuyas consecuencias las sufren los países vecinos, como el mismo Japón.

\section{Relaciones políticas}

Las relaciones económicas son cada vez más fuertes entre China y Japón, tanto que en una reunión a fines de mayo del 2010 en Jeju, Corea del Sur, entre este país, China y Japón, el Primer Ministro chino, Wen Jiabao, pidió que se elaborara un estudio de factibilidad para que haya un Tratado de Libre Comercio (TLC) entre los tres países, estudio que debería estar terminando para el $2012^{20}$. Después de esa visita el Primer Ministro chino fue a Japón para una estadía de tres días.

Las relaciones entre China y Japón han pasado por periodos complicados. Hace casi 2000 años algunos japoneses empezaron a viajar a China a aprender muchas cosas del país más civilizado de la Tierra en ese entonces, y esto se fortaleció 500 años después con visitas incluso de funcionarios del gobierno japonés a China. De allí Japón aprendió la escritura, el confucianismo, su forma de gobierno, el budismo (a través de Corea), que los japoneses adaptaron en forma inteligente a las condiciones propias de su país.

Los dos países se aislaron del mundo desde comienzos del siglo XVII mientras Occidente iba a empezar después un periodo de modernización que les permitiría pronto convertirse en las potencias coloniales que van a invadir la región asiática. Pero a fines del siglo XIX Japón empieza a modernizarse, antes que China, y en 1894-5 le hace una guerra y ocupa Taiwán. Luego, en el siglo pasado, en las décadas de 1930 y 1940 ocurre la invasión japonesa a China, hasta la derrota de Japón en la II Guerra Mundial en 1945.

20 "Chinese Premier makes four-point proposal on deepening economic cooperation with S. Korea, Japan", http://news.xinhuanet.com/english2010/china/2010-05/30/c_13323439.htm 


\section{Relaciones Económicas China-Japón: Los dos gigantes de Asia cada vez más integrados}

Después de eso las relaciones entre estos dos países estuvieron interrumpidas, pero en 1972 Japón otra vez establece relaciones diplomáticas con China. Desde ese momento las relaciones económicas entre los dos países crecieron, y sus relaciones políticas también, aunque estas últimas se enfriaron cuando algunos políticos japoneses visitaron, a pesar de las protestas de China, un templo sintoísta, el Yasukuni, donde están enterrados algunos jefes militares japoneses que se consideran fueron criminales de guerra. Esto sucedió especialmente durante el gobierno del Primer Ministro japonés Junichiro Koizumi, entre el 2001 y el 2005.

En marzo del 2005 hubo manifestaciones antijaponesas en algunas ciudades de China, como en Beijing y Shangai, en protesta ante la aprobación por parte del gobierno japonés de la publicación de unos libros de historia, que según los chinos ocultaba el rol que Japón jugó en la invasión que hizo a China en los años 1930 y comienzos de 1940 y minimizaba las atrocidades que el país nipón cometió en ese periodo.

Por ahora China y Japón tienen intereses comunes, como el desarrollo económico, donde China necesita la tecnología, el capital y los conocimientos empresariales de Japón, mientras este país necesita la mano de obra barata china para producir bienes para el mundo y sobre todo para vender al inmenso mercado chino de 1350 millones de consumidores, que mejoran rápidamente su poder adquisitivo.

Además, también está presente el tema de la seguridad regional, donde chinos y japoneses necesitan colaborar, especialmente dada la volátil situación en la península coreana, donde Corea del Norte, un estado ermitaño con un gobierno que mantiene a más de un millón de soldados en armas mientras la mayoría de su población se muere literalmente de hambre, y que tiene bombas atómicas a su disposición, toma una actitud cada vez más beligerante. China es el único país que puede influir en Corea del Norte, pues le brinda apoyo económico y militar

Entre los temas que podrían enturbiar las relaciones entre China y Japón se menciona el asunto del pasado histórico reciente, la disputa territorial por unas islas al sur de Japón, y la competencia por recursos naturales que los dos países necesitan conseguir del mundo. En todo caso, esos factores, más la desconfianza entre ambos países, son los que aún impiden una mayor relación, como lo menciona una reciente encuesta hecha 


\section{Carlos Aquino Rodríguez}

en ambos países ${ }^{21}$, pero ahí mismo se menciona que estas relaciones tienden a mejorar en el futuro.

En el plano internacional, la presencia de China tiende a crecer y la de Japón a disminuir. Por ejemplo, Japón en la Conferencia Internacional de Partidos Políticos en Asia (ICAPP, por sus siglas en inglés) manifestó que ya no podrá dar más ayuda económica debido a los grandes problemas fiscales que tiene ${ }^{22}$. En la misma Conferencia se destacó el creciente rol de China como donante en la región.

\section{Conclusiones}

Las relaciones económicas entre los dos colosos del Asia cada vez son mayores. China es cada vez más importante para Japón, como destino de sus exportaciones, como fuente de sus importaciones, como destino de su inversión extranjera. La mano de obra barata china hace que ese país sea usado por las empresas japonesas como base para producir para el mundo (aunque últimamente los salarios en China han subido y en junio y julio del 2010 hubo huelgas en demanda de mayores remuneraciones ${ }^{23}$ ). Pero también las empresas japonesas se instalan ahí para producir cada vez más para el propio mercado chino, que tiene el mayor número de consumidores en el mundo, que cada vez incrementan su nivel de ingreso.

China también es importante, pues Japón necesita consumidores para sus productos. Una forma, aparte de las ventas que las compañías japonesas hacen a China a través de las exportaciones, es el turismo de los chinos que visitan Japón. Se calcula que para el 2020 habrá 10 millones de turistas chinos en Japón, y turistas ricos, que gastan más que cualquier otro turista. Japón también necesita trabajadores chinos para sus fábricas, y cada vez vienen más de ellos, pues la población japonesa disminuye y su mano de obra ya es cara (y tampoco quieren trabajar en las fábricas en labores intensivas en mano de obra).

Por el lado de China, también la relación con Japón es importante, pues necesita

21 "Asian neighbors' ties warm", http://www.chinadaily.com.cn/china/2010-08/16/content_11155366.htm

22 "Japan Suffering From "Aid Fatigue"”, http://news.ino.com/headlines/?newsid=71920101759

23 "The rising power of the Chinese worker", The Economist, Julio 29, 2010, http://www.economist.com/ node/16693333 


\section{Relaciones Económicas China-Japón: Los dos gigantes de Asia cada vez más integrados}

especialmente la tecnología, el capital y el conocimiento empresarial nipón. Y los chinos quieren aprender lo último también yendo a Japón, adquiriendo empresas ahí. Así que la inversión china aumentará en Japón.

Como los gigantes en el Asia Oriental, China y Japón serán el eje del mercado asiático cada vez más integrado, y cuyo dinamismo económico continuará en el futuro. Países como el Perú, que ya tiene un TLC con China y agosto 2010 otro con Japón (y también con Corea del Sur) debemos prestarle más atención.

\section{BIBLIOGRAFÍA}

Aquino, Carlos (2009). "Cambio de Gobierno en Japón y sus efectos en la economía". Revista Calidad y Creatividad. Facultad de Ciencias Económicas y Empresariales, Universidad Ricardo Palma, diciembre del 2009, pp. 36-47.

(2010). "La revaluación del yuan y sus efectos". Revista Calidad y Crea-

tividad. Facultad de Ciencias Económicas y Empresariales, Universidad Ricardo Palma, julio del 2010, pp.91-97

(2010). “¿Puede Japón ser otra Grecia? El peso de la deuda pública y los problemas de la economía japonesa”, julio del 2010, http://economia.unmsm.edu. pe/ (Ver Artículos y Noticias).

Ministry of Foreign Affairs of Japan: "Japan's ODA White Paper 2009-Japan International Cooperation-", http://www.mofa.go.jp/policy/oda/white/2009/index.html 